\title{
Mise en évidence d' $\alpha$-lactalbumine glycosylée dans une préparation industrielle d' $\alpha$-lactalbumine bovine
}

\author{
J.J. Baumy et J. Fauquant
}

Laboratoire de recherche de technologie laitière, INRA, 65, rue de Saint-Brieuc, 35042 Rennes Cedex, France

(reçu le 18 janvier 1989; accepté le 3 avril 1989)

\begin{abstract}
Résumé - Ce travail met en évidence la présence d' $\alpha$-lactalbumine glycosylée dans une préparation industrielle d' $\alpha$-lactalbumine bovine. Les protéines sont séparées par chromatographie de gel filtration sur Sephadex G 50 (Pharmacia) puis ultérieurement identifiées par HPLC. La séparation a permis d'obtenir 4 fractions dont les rapports pondéraux ont été déterminés. La présence d' $\alpha$-lactalbumine glycolysée a été mise en évidence par des dosages d'acides aminés et par des électrophorèses révélant les glycoprotéines. Une évaluation montre qu'environ $7 \%$ de l' $\alpha$-lactalbumine est glycosylée dans cette préparation.
\end{abstract}

$\alpha$-lactalbumine - glycoprotéine - gel filtration

Summary - Evidence for the presence of glycosylated $\alpha$-lactalbumin in an industrial bovine $\alpha$-lactalbumin preparation.. This work demonstrates the presence of glycosylated $\alpha$-lactalbumin in an industrial bovine $\alpha$-lactalbumin preparation. The proteins were separated using Sephadex $G 50$ (Pharmacia) gel filtration chromatography, then characterized using HPLC. The protein contents of the 4 fractions were calculated. Glycosylated $\alpha$-lactalbumin was characterized by amino-acids composition and electrophoresis with glycoprotein staining. The amount of glycosylated $\alpha$-lactalbumin was about $7 \%$ of total $\alpha$-lactalbumin.

$\alpha$-lactalbumin - glycoprotein - gel filtration

\section{INTRODUCTION}

Le lactosérum est un sous-produit laitier, contenant des protéines, qui peut être facilement valorisé à des fins nutritionnelles ou technologiques. Dans le but de préparer des laits maternisés, l' $\alpha$-lactalbumine est séparée de la $\beta$-lactoglobuline par agrégation et précipitation sous l'ac- tion combinée d'un abaissement de $\mathrm{pH}$ et d'un traitement thermique (Pearce, 1983; Pierre \& Fauquant, 1986). Cette précipitation entraîne, outre l' $\alpha$-lactalbumine, d'autres protéines solubles de poids moléculaire élevé (Sérum Albumine Bovine (SAB), immunoglobulines, etc.), qu'il est possible d'éliminer par tamisage moléculaire (Pierre \& Fauquant, 1986). II a été 
montré, par chromatographie de gel filtration, que l' $\alpha$-lactalbumine contenue dans de telles préparations était hétérogène (Baumy \& Brulé, 1988); ce phénomène a aussi été observé dans des préparations commerciales (Kim \& Kim, 1986).

L' $\alpha$-lactalbumine bovine, de poids moléculaire 14200 , possède 2 variants génétiques $A$ et $B$, se différenciant par un acide aminé; le variant normalement présent dans les troupeaux laitiers européens est le variant B (Lyster, 1972). Chaque variant étudié en chromatographie d'échange d'ions, présente 2 pics qui ne proviennent pas d'une hétérogénéité, mais de l'interaction de la protéine avec les ions $\mathrm{NH}_{4}{ }^{+}$ou $\mathrm{SO}_{4}{ }^{2-}$ - du tampon; le rapport de surface des 2 pics dépend des traitements subis lors de la préparation de l'échantillon (Hopper, 1973). Ce comportement chromatographique, qui n'existe pas pour les protéines de lait de toutes les espèces de mammifères (Hopper \& McKenzie, 1974), se retrouve dans I' $\alpha$-lactalbumine humaine (Prieels \& Schlusselberg, 1977).

Outre cette particularité, l' $\alpha$-lactalbumine bovine possède 2 formes, dont une glycosylée (Hindle \& Wheelock, 1970) comportant 11 à 12 résidus hexoses et présentant une mobilité électrophorétique en milieu alcalin, inférieure à celle de l' $\alpha$-lactalbumine majeure; les deux protéines ont une activité équivalente dans le système enzymatique de la lactose synthétase (Barman, 1970). Cet auteur a ultérieurement séparé 3 formes d' $\alpha$-lactalbumine, dont une glycolysée (Barman, 1972). D'autres travaux ont montré la présence, en plus de la protéine majeure, de 3 composants nommés $S_{1}, S_{2}$ et $F$ (Hopper \& McKenzie, 1973), ayant des propriétés immunologiques équivalentes (Préaux et al., 1965); seuls les composants $S_{1}$ et $S_{2}$ sont glycosylés. La composition en carbohydrates diffère entre les composants et selon les auteurs, mais on note la présence d'hexoses, d'hexosa- mines, de désoxyhexoses et d'acide sialique (Barman, 1970; Hopper \& McKenzie, 1973; Prasad et al., 1980). La liaison de ces résidus sur la chaîne protéique se réalise au niveau de Asn 45 (Hopper \& McKenzie, 1973).

Des composants mineurs ont aussi été mis en évidence dans l' $\alpha$-lactalbumine de chienne, de babouine, de lapine (Quarfoth \& Jenness, 1975), de rate (Prasad et al., 1980). Chez la souris, 5 à $10 \%$ de l' $\alpha$-lactalbumine est glycosylée et présente des propriétés immunologiques et une activité de lactose synthétase équivalentes à celles de l' $\alpha$-lactalbumine majeure (Nagamatsu \& Oka, 1980).

L'utilisation d' $\alpha$-lactalbumine en alimentation infantile nécessite l'obtention d'une protéine de grande pureté. Le produit obtenu par la méthode de Pierre \& Fauquant (1986), étant hétérogène, ne répond pas à cette exigence. L'objet de ce travail est de mettre en évidence la présence d' $\alpha$-lactalbumine glycosylée dans une préparation industrielle d' $\alpha$-lactalbumine et de la quantifier.

\section{MATÉRIEL ET MÉTHODES}

La préparation d' $\alpha$-lactalbumine provient d'un essai pilote industriel. La précipitation de l' $\alpha$-lactalbumine était réalisée à partir de lactosérum de caséinerie, selon la méthode décrite par Pierre \& Fauquant (1986); le précipité était séparé par microfiltration puis lavé par diafiltration. Après resolubilisation en milieu basique, l' $\alpha$-lactalbumine était lyophilisée.

\section{Déterminations analytiques}

\section{Chromatographie de gel filtration à basse pression}

La séparation des différents constituants protéiques de la poudre, après solubilisation (200 g..$\left.^{-1}\right)$ dans le tampon Tris/ $\mathrm{HCl}$, a été réali- 
sée par chromatographie de gel filtration.

Colonne : $L=140 \mathrm{~cm}$ - Diamètre $=4,5 \mathrm{~cm}$;

gel : Sephadex G 50 (Pharmacia);

tampon : Tris $0,02 \mathrm{M}-\mathrm{HCl}-\mathrm{pH} 7,5$;

Système de chromatographie basse pression : Pharmacia;

échantillon : $1 \mathrm{~g}$ de poudre pour $5 \mathrm{ml}$ de tampon;

débit : $25 \mathrm{ml} \cdot \mathrm{h}^{-1}$;

durée : $17 \mathrm{~h}$;

température : ambiante;

volume des fractions ; $4,5 \mathrm{ml}$;

détection : $280 \mathrm{~nm}$.

\section{Dosage des protéines}

La séparation et le dosage des protéines ont été réalisés en chromatographie de gel filtration HPLC (Varian 5000) sur colonne Micropak G $300 \mathrm{SW}(30 \mathrm{~cm}$ - Varian) avec un tampon Tris $/ \mathrm{HCl}\left(0,01 \mathrm{M}, \mathrm{pH} 6,68\right.$, débit $1 \mathrm{ml} \cdot \mathrm{min}^{-1}$. $280 \mathrm{~nm}$ ). La précision de la méthode est de $5 \%$. Les standards utilisés étaient des protéines (type III, Sigma Chemical Company); les étalons étaient préparés par pesée en tenant compte de leur pureté.

\section{Dosage des acides aminés}

Le dosage des acides aminés était réalisé en double selon le protocole de Spackman et al. (1958) sur Amino Acid Analyzer Beckman (hydrolyse à $110^{\circ} \mathrm{C}$ pendant $24 \mathrm{~h}$ ).

\section{Dosage d'azote}

Le dosage d'azote était effectué par la méthode Kjeldhal sur système Kjeltec System 1003 (Tecator).

\section{Détermination de l'extrait sec}

L'échantillon (environ $0,5 \mathrm{~g}$ de poudre) était déshydraté à $105^{\circ} \mathrm{C}$ dans une étuve pendant $7 \mathrm{~h}$.

\section{Détermination de la teneur en cendres}

L'échantillon était minéralisé pendant $3 \mathrm{~h} 30$ à $530-550^{\circ} \mathrm{C}$.

\section{Electrophorèses}

La séparation des protéines a été réalisée sur gel de polyacrylamide à $\mathrm{pH} 8,3$, sans SDS et sans urée, selon le protocole décrit par
Andrews (1983). La coloration des glycoprotéines a été effectuée selon la méthode de Kapitany \& Zebrowski (1973). La liaison C-C des groupements $\alpha$-glycol est oxydée par l'acide périodique pour donner un dialdéhyde qui réagit avec le réactif de Schiff pour donner un composé coloré. $150 \mu \mathrm{g}$ d'échantillon étaient déposés dans chaque puits.

\section{RÉSULTATS}

\section{Composition des poudres}

L'extrait sec de la poudre est de 980,9 g. $\mathrm{kg}^{-1}$; sa teneur en protéines ( $\mathrm{N} \mathrm{x}$ 6,38 ) est de $917,6 \mathrm{~g} \cdot \mathrm{kg}^{-1}$; la teneur en cendres est égale à $29,0 \mathrm{~g} \cdot \mathrm{kg}^{-1}$. La teneur en $\alpha$-lactalbumine déterminée par HLPC

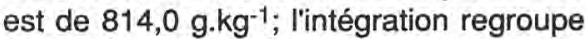
l' $\alpha$-lactalbumine et l'épaulement qui le précède; cet épaulement correspond à la protéine que nous devons caractériser.

\section{Séparation des différents constituants par chromatographie}

La chromatographie de gel filtration à basse pression de l'échantillon a permis de séparer 4 pics. Le chromatogramme correspondant est présenté à la Figure 1. L'identification des 4 pics est réalisée par gel filtration en HPLC par rapport à des étalons témoins (Fig. 2) et par électrophorèse. Le premier pic correspond à des composés de poids moléculaire élevé; le second pic renferme la $\mathrm{SAB}$. Le troisième pic regroupe la protéine indéterminée que nous devons caractériser, de l' $\alpha$-lactalbumine et des traces de $\mathrm{SAB}$. Le quatrième pic correspond à l' $\alpha$-lactalbumine majeure. 
Les collectes correspondant aux 4 pics ont été regroupées en 4 fractions dont l'extrait sec a été déterminé sur une partie aliquote. Après correction, tenant compte de l'extrait sec du tampon, la répartition pondérale de chaque fraction a été déterminée. Les fractions $1,2,3$ et 4 renferment respectivement $16,9,3,7,9,7$, et $69,7 \%$ du poids total des protéines éluées dans ces fractions.

Pour la fraction 3 , la surface intégrée par HLPC correspondant au composé indéterminé représente $59 \%$ de la surface totale intégrée (Fig. 2).

\section{Comparaison des fractions 3 et 4}

Les compositions en acides aminés (sauf cystéine) des fractions 3 et 4 sont données dans le Tableau I. Les coefficients de corrélation entre la composition donnée par l'analyse et la composition théorique de l' $\alpha$-lactalbumine (Eigel et al., 1984 ) sont de 0,986 et 0,991 , pour les fractions 3 et 4 .

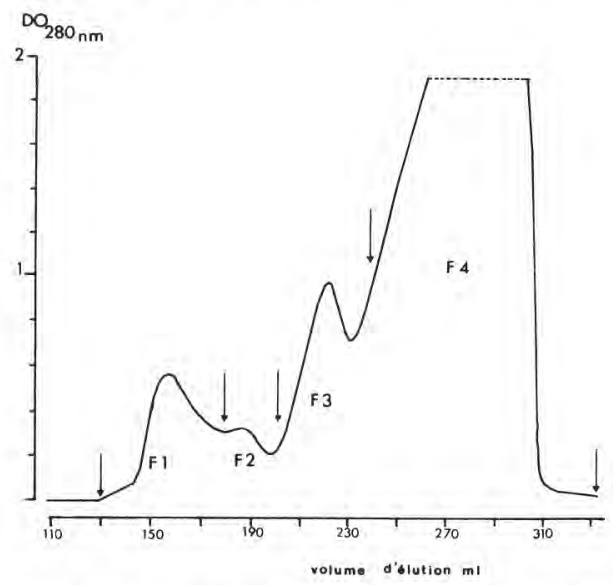

Fig. 1. Profil chromatographique de la séparation en gel filtration (Sephadex G50 - Pharmacia) de l'échantillon d' $\alpha$-lactalbumine. Tampon Tris $0,02 \mathrm{M} / \mathrm{HCl} \mathrm{pH} 7,5$. Les flèches indiquent le début et la fin de chaque fraction (F) : F1, $F 2, F 3$ et F4.

\section{Etude de la fraction 3}

Pour confirmer le résultat précédent, nous avons réalisé un second fractionnement par chromatographie de gel filtration de l'échantillon de départ et avons déterminé la composition en acides aminés de différentes fractions individuelles d'éluats de $4,5 \mathrm{ml}$. Les compositions en acides aminés sont données dans le Tableau II. Les coefficients de corrélation vis-à-vis de la composition de l' $\alpha$-lactalbumine (Eigel et al., 1984) et les surfaces relatives des 2

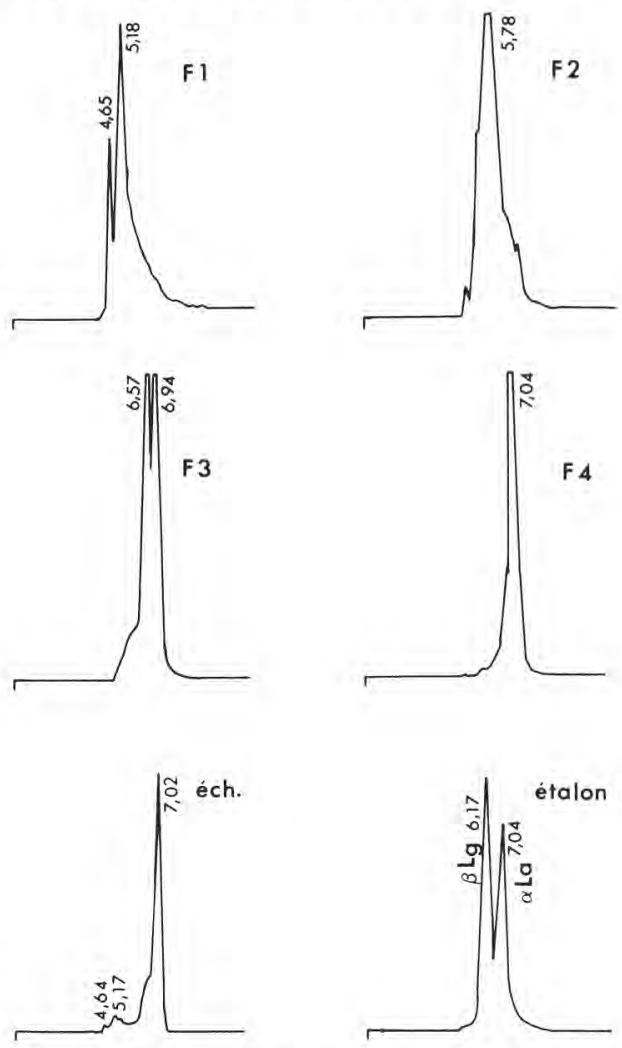

Fig. 2. Profil chromatographique HPLC (gel filtration sur colonne Micropak G 300 SW, tampon Tris $0,01 \mathrm{M} / \mathrm{HCl} \mathrm{pH} 6,68$ ) de la préparation d' $\alpha$ lactalbumine (éch.), de ses fractions F1, F2, F3 et F4 séparées par gel filtration (Sephadex G50 - Pharmacia) et d'un étalon d' $\alpha$-lactalbumine $(\alpha$ La) et de $\beta$-lactoglobuline $(\beta \mathrm{Lg})$. 
Tableau I. Teneur en acides aminés (g pour $100 \mathrm{~g}$ d'acides aminés totaux) des fractions 3 (F3) et 4 (F4) séparées par chromatographie en gel filtration (Sephadex G50 - Pharmacia) et composition théorique de l' $\alpha$-lactalbumine d'après Eigel et al. (1984).

\begin{tabular}{lrrr}
\hline & $F 3$ & $F 4$ & $\alpha$-lactalbumine \\
& & & \\
\hline Asp & 18,4 & 18,7 & 18,4 \\
Thr & 5,6 & 5,4 & 5,5 \\
Ser & 5,1 & 5,0 & 4,8 \\
Glu & 14,2 & 14,4 & 16,5 \\
Pro & 3,2 & 3,1 & 1,5 \\
Gly & 2,8 & 2,9 & 3,0 \\
Ala & 2,1 & 1,9 & 1,8 \\
Val & 4,7 & 4,7 & 4,6 \\
Met & 1,1 & 1,1 & 1,0 \\
Ile & 6,7 & 7,1 & 6,9 \\
Leu & 12,3 & 11,5 & 11,2 \\
Tyr & 4,3 & 4,4 & 4,8 \\
Phe & 4,6 & 4,4 & 4,3 \\
His & 3,2 & 3,2 & 3,1 \\
Lys & 9,8 & 10,6 & 11,5 \\
Arg & 1,8 & 1,7 & 1,1 \\
& & & \\
\hline
\end{tabular}

pics, déterminées par HPLC, sont rassemblés dans le Tableau III. Ces coefficients de corrélation valent 0,987 ou 0,988 , quel que soit le rapport de surface des 2 pics correspondant à la protéine indéterminée et à l' $\alpha$-lactalbumine.

Les électrophorèses, selon Andrews (1983), présentées à la Figure 3 (A) montrent, pour ces fractions individuelles, trois bandes distinctes. La bande qui migre le plus correspond à l' $\alpha$-lactalbumine majeure. Les 2 autres bandes, qui migrent moins rapidement, correspondent à la protéine indéterminée; elles ont une charge apparente inférieure à celle de l' $\alpha$-lactalbumine. La coloration spécifique des protéines glycosylées (Fig. $3(B)$ ) montre 2 bandes situées au même niveau que les protéines indéterminées; leur intensité décroît lorsque la teneur en $\alpha$-lactalbumine majeure augmente.

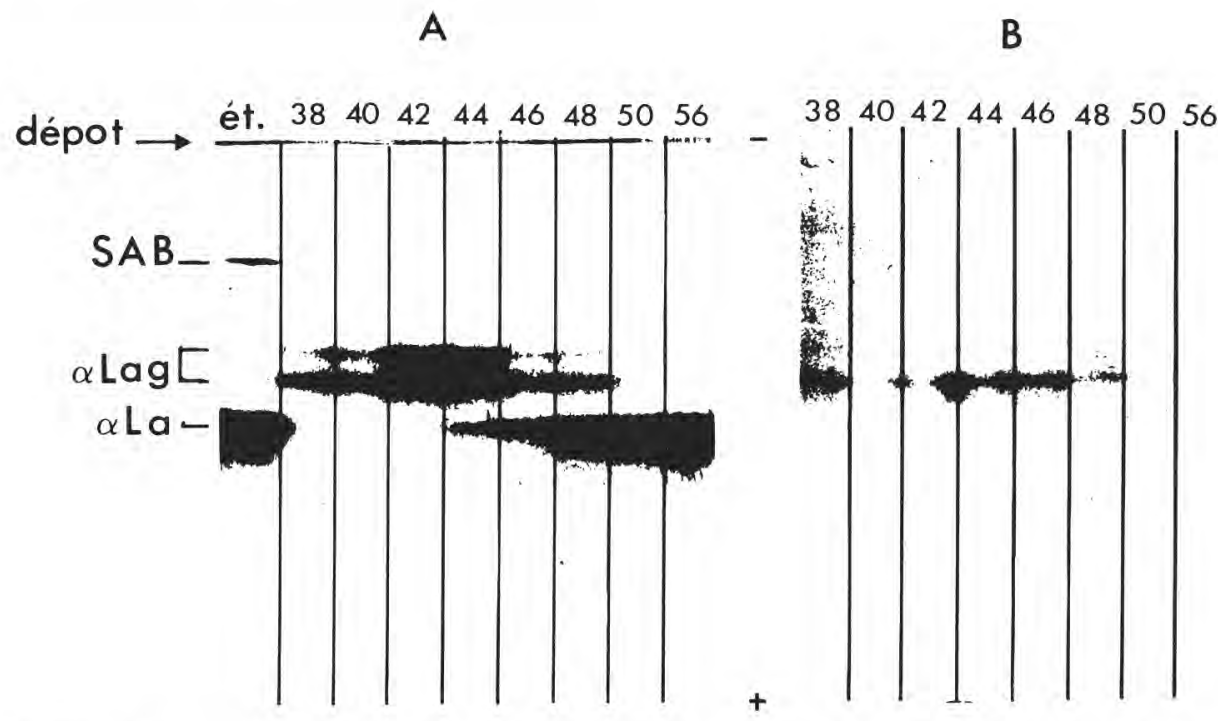

Fig. 3. Electrophorèses selon Andrews (1983) (A) révélant les protéines et selon Kapitany \& Zebrowski (1973) (B) révélant les glycoprotéines contenues dans un étalon (ét.) et dans les tubes $38,40,42,44,46,48,50$ et 56 de la séparation par gel filtration (Sephadex G50 - Pharmacia). (SAB $=$ Serum Albumine Bovine; $\alpha \mathrm{La}=\alpha$-lactalbumine; $\alpha \mathrm{Lag}=\alpha$-lactalbumine glycosylée) . 
Tableau II. Teneur en acides aminés (g pour $100 \mathrm{~g}$ d'acides aminés totaux) des protéines contenues dans les tubes $40,44,46$ et 56 séparés par gel filtration (Sephadex G50 - Pharmacia) et de l' $\alpha$-lactalbumine (Type III - Sigma).

\begin{tabular}{lrrrrr}
\hline & $T 40$ & $T 44$ & $T 46$ & $T 56$ & $\alpha$-lactalbumine \\
\hline & & & & & \\
Asp & 16,5 & 17,7 & 18,2 & 18,9 & 19,4 \\
Thr & 5,4 & 5,1 & 4,5 & 5,5 & 5,3 \\
Ser & 5,1 & 5,0 & 4,8 & 4,7 & 4,8 \\
Glu & 14,6 & 13,9 & 13,8 & 13,5 & 13,3 \\
Pro & 2,8 & 2,7 & 3,0 & 2,6 & 2,3 \\
Gly & 2,8 & 2,9 & 2,9 & 3,0 & 3,0 \\
Ala & 2,1 & 2,0 & 2,0 & 1,9 & 1,8 \\
Val & 4,4 & 4,6 & 4,6 & 4,5 & 4,2 \\
Met & 0,8 & 0,8 & 1,3 & 1,2 & 1,1 \\
lle & 8,5 & 8,3 & 8,1 & 6,7 & 11,9 \\
Leu & 11,7 & 11,7 & 11,5 & 11,6 & 5,0 \\
Tyr & 4,1 & 4,3 & 4,4 & 4,7 & 4,5 \\
Phe & 4,2 & 4,3 & 4,4 & 4,4 & 3,4 \\
His & 3,2 & 3,3 & 3,3 & 3,3 & 12,0 \\
Lys & 11,7 & 11,8 & 11,7 & 11,8 & 1,3 \\
Arg & 1,7 & 1,6 & 1,5 & 1,3 & \\
& & & & & \\
\hline
\end{tabular}

\section{DISCUSSION}

Les méthodes de séparation de l' $\alpha$-lactalbumine par chromatographie de gel filtration ont été choisies, car elles permettaient d'éviter le dédoublement du pic

Tableau III. Coefficients de corrélation ( $r$ ) entre composition en acides aminés des protéines des tubes $40,44,46,56$ et composition de l' $\alpha$ lactalbumine (d'après Eigel et al., 1984). Surfaces du pic indéterminé et du pic d' $\alpha$-lactalbumine intégrés par HPLC (unités arbitraires).

\section{$r$ Surface Surface pic $\quad \alpha$-lactal- indéterminé bumine}

\begin{tabular}{lrrr} 
Tube 40 & 0,988 & 176 & 96 \\
Tube 44 & 0,987 & 142 & 127 \\
Tube 46 & 0,987 & 133 & 155 \\
Tube 56 & 0,987 & 0 & 503 \\
$\begin{array}{l}\alpha \text {-lactalbumine } \\
\text { (Sigma) }\end{array}$ & 0,984 & 0 & 150 \\
\hline
\end{tabular}

correspondant à cette protéine lors de la séparation par échange d'ions (Hopper, 1973).

Les résultats de la répartition pondérale des 4 fractions isolées par chromatographie montrent qu'environ $21 \%$ du produit correspond à des protéines autres que l' $\alpha$-lactalbumine. En additionnant les teneurs correspondant aux fractions 3 et 4 , on trouve une valeur de $79,4 \%$, équivalente à celle donnée par HPLC, qui englobe l' $\alpha$-lactalbumine et la protéine contaminante $(81,4 \%)$. La fraction 3 n'est pas pure; la surface d'intégration relative à la protéine indéterminée ne représente que $59 \%$ de la surface totale. La composition en acides aminés de cette fraction est semblable à celle de l' $\alpha$-lactalbumine (Eigel et al., 1984). Les coefficients de corrélation entre composition déterminée par l'analyse et composition théorique sont équivalents pour les deux fractions 3 et 4 ce qui tend à prouver que le composé indéterminé est de l' $\alpha$-lactalbumine. 
Ce résultat est confirmé par une étude de la composition en acides aminés des protéines contenues dans les tubes de la fraction 3 collectés individuellement; leur composition est équivalente à celle de l' $\alpha$-lactalbumine. La mise en évidence de résidus glycosylés sur ces protéines permet de conclure que la protéine indéterminée correspond à différentes formes de glycosylation de l' $\alpha$-lactalbumine. Les 2 bandes relatives à des protéines glycosylées détectées en électrophorèse et ayant une migration plus lente que l' $\alpha$-lactalbumine majeure correspondraient aux composants $S_{1}$ et $S_{2}$ décrits par Hopper \& McKenzie (1973).

En HPLC, nous n'avons pas totalement séparé ces 3 composants. Le poids moléculaire (PM) de ces protéines est plus élevé que celui de l' $\alpha$-lactalbumine majeure. En considérant les temps de rétention en HLPC de la $\beta$-lactoglobuline (PM $=18400$ ) et de l' $\alpha$-lactalbumine, respectivement égaux à 6,17 et 7,04 , et la relation linéraire existant entre $\log (\mathrm{PM})$ et le temps de rétention, il est possible de déterminer le PM de l' $\alpha$-lactalbumine glycosylée (temps de rétention $=6,57$ ), qui est égal à $16300 \pm 400$; la partie glucidique représente environ $13 \%$ du poids de la protéine. Ces valeurs sont en accord avec celles de Barman (1970), respectivement égales à 16800 pour la première et à $15 \%$ pour la seconde.

En supposant que les coefficients d'absorption molaire de l' $\alpha$-lactalbumine et de l' $\alpha$-lactalbumine glycosylée sont équivalents, l'intégration par HPLC de la fraction 3 donne $59 \%$ d' $\alpha$-lactalbumine glycosylée. Les résultats de la répartition pondérale montrent donc que $7,2 \%$ (poids/poids) de l' $\alpha$-lactalbumine est glycosylée, ce qui correspond à 1,0\% (poids/poids) de sucres. Nos résultats sont supérieurs à ceux de Hopper et McKenzie (1973), qui ont montré que les composants $S_{1}$ et $S_{2}$ ne représentaient que $3 \%$ de l' $\alpha$-lactalbumine.

Nous avons mis en évidence, dans une préparation industrielle d' $\alpha$-lactalbumine, la présence de cette protéine sous forme glycolysée. Les 2 types d' $\alpha$-lactalbumine ont des propriétés physico-chimiques proches qui les rendent difficilement séparables. Des travaux ultérieurs devront confirmer la composition exacte en carbohydrates et comparer les propriétés physico-chimiques de ces 2 protéines.

\section{REMERCIEMENTS}

Nous tenons à remercier M. Piot pour sa participation aux analyses d'acides aminés.

\section{RÉFÉRENCES}

Andrews A.T. (1983) Proteinases in normal bovine milk and their action on caseins. $J$. Dairy Res. 50, 45-55

Barman T.E. (1970) Purification and properties of bovine milk glyco- $\alpha$-lactalbumin. Biochim. Biophys. Acta 214, 242-244

Barman T.E. (1972) The modification of the tryptophan residues of bovine $\alpha$-lactalbumin with 2-hydroxy-5-nitrobenzyl bromide and with dimethyl (2-hydroxy-5-nitrobenzyl) sulphonium bromide. I. Characterization of the modified protein. Biochim. Biophys. Acta 258, 297-313

Baumy J.J. \& Brulé G. (1988) Binding of bivalent cations to $\alpha$-lactalbumin and $\beta$-lactoglobulin : effect of $\mathrm{pH}$ and ionic strength. Lait 68, 3348

Eigel W.N., Butler J.E., Ernstrom C.A., Farrell H.M., Harwalkar V.R., Jenness R. \& Withney R. McL. (1984) Nomenclature of protein of cow's milk: fifth revision. J. Dairy Sci. 67, 1599-1631

Hindle E.J. \& Wheelock J.V. (1970) Carbohydrates of bovine $\alpha$-lactalbumin preparations. Biochem. J. 119, 14P 
Hopper K.E. (1973) Apparent heterogeneity of bovine $\alpha$-lactalbumin A and B in DEAE-Sephadex chromatography. Biochim. Biophys. Acta 293, 364-370

Hopper K.E. \& McKenzie H.A. (1973) Minor components of bovine $\alpha$-lactalbumin A and B. Biochim. Biophys. Acta 295, 352-363

Hopper K.E. \& McKenzie H.A. (1974) Comparative studies of $\alpha$-lactalbumin and lysozyme: Echidna lysozyme. Mol. Cell. Chem. 3, 93-108

Kapitany R.A. \& Zebrowski E.J. (1973) A high resolution PAS stain for polyacrylamide gel electrophoresis. Anal. Biochem. 56, 361-369

Kim J. \& Kim H. (1986) Fusion of phospholipid vesicles induced by $\alpha$-lactalbumin at acidic $\mathrm{pH}$. Biochemistry 25, 7867-7874

Lyster R.L.J. (1972) Reviews of the progress of dairy science. Section C - Chemistry of milk proteins. J. Dairy Res. 39, 279-318

Nagamatsu Y. \& Oka T. (1980) Purification and characterization of mouse $\alpha$-lactalbumin and preparation of its antibody. Biochem. J. 185, 227-237

Pearce R.J. (1983) Thermal separation of $\beta$-lactoglobulin and $\alpha$-lactalbumin in bovine cheddar cheese whey. Aust. J. Dairy Technol. 33, 144-149

Pierre A. \& Fauquant J. (1986) Principes pour un procédé industriel de fractionnement des protéines de lactosérum. Lait 66, 405-419

Prasad R., Hudson B.G., Strickland D.K. \& Ebner K.E. (1980) The structure of the asparagine-linked carbohydrate unit of rat $\alpha$-lactalbumin. J. Biol. Chem. 255, 1248-1251

Préaux G., De Weer P., Peirsman E., Tielemans K. \& Lontie R. (1965) Concentration à partir du sérum de lait de 3 composants immunologiquement identiques à l' $\alpha$-lactalbumin. Arch. Int. Physiol. Biochim. 73, 154-155

Prieels J.P. \& Schusselberg J. (1977) Heterogeneity in $\alpha$-lactalbumin - I - Human $\alpha$-lactalbumin. Biochim. Biophys. Acta 491, 76-81

Quarfoth G.J. \& Jenness R. (1975) Isolation, composition and functional properties of $\alpha$-lactalbumins from several species. Biochim. Biophys. Acta 379, 476-487

Spackman D.H., Stein W.H. \& Moore S. (1958) Automatic recording apparatus for use in the chromatography of amino-acids. Ann. Chem. 30, 1190-1206 